\title{
SONGS OF THE SPANISH JEWS IN THE BALKAN PENINSULA. ${ }^{1}$
}

\author{
VIII. ${ }^{2}$
}

Arvolēra, mi arvolēra, Tan galana i tan džentil:

La rais tyēne di oru

I la rama di marfil.

En la ramika mas čika

Sta la dama tan džentil,

Peñandusi lus sus kaveyus

Kon un peñi di marfil.

Por ayi pasó un kavayēru

Kē asimežava ${ }^{3}$ a Amadi:

"Asi bivas, kavayēru,

Si vistiš a Amadi ?"

"Byen lu vidi i lu konosku,-

Letra mandava kon mi !"

"Kwantu dyēraš, la mi siñora,

Kē volu trušēra aki ?"

"Dyēra yō lus trēs mil dukadus

Kẽ mi kedarun di Amadi."

"Mas dyēraš, la mi siñora,

Kē volu trušēra aki !"

"Dyēra yō lus trēs mulinus

Kē mi kedarun di Amadi:

El unu muēli kanēla

I el otru muēli džinfil; ${ }^{4}$

El mas čikitiku di eyus

Muēli arina para Amadi."

"Mas dyēraš, la mi siñora,

Kē volu trušēra aki!"

"Dyēra yō lus mis trēnsadus

Kon mi peñi di marfil.”

"Mas dyëraš, la mi siñora,

Kē volu trušēra aki !"

"Dyēra yō las trēs fižikas ${ }^{5}$

Kề mi kedarun di Amadi:

1 Concluded from Vol. I, No. 1, pp. 205-16.

2 Another version is given in Danon's collection. ${ }^{3} \mathrm{~S}$. semejar. ${ }^{4}$ "Clove." ${ }^{5} \mathrm{~S}$. hijica.

[Modern PhILOLOGY, October, 1903 
La una meti la meza

I la otra para servir;

La mas čikitika di eyas

Para folgar i dormir."

"Dyēraš vos el vwestru kuerpu

Kē volu trušēra aki ?"

"A mal vaya el kavayēru

Kē estu fue a dezir!"

"No vus aravyeš, la mi siñora,

Kē yō so vwestru maridu Amadi !"

"Una vēs kē soš ${ }^{1}$ mi maridu,

Kē siñal davaš a mi ?"

"Debašo del peču syerdu ${ }^{2}$

Un kavēyu vos akušaka ${ }^{3}$ a mi."

\section{IX. ${ }^{4}$}

Mi padri ēra di Brusa

Mi madri di Aragon:

Kazarunsi endžuntus, ${ }^{5}$

Nasyērami yō.

Mihamá ${ }^{6}$ kē ēra regalada

En čika mi kazó,

Dyēra mi por maridu

A un riku pastor.

Al vēz di kazadu

Su vertud amostró;

El si komia la karni,

Lus gwēsizikus ${ }^{7}$ yō;

El si komia el pan blanku,

I el morēnu yō;

El si komia el pēši

I las espinikas yō;

El si bevia el vinu,

I la agwa yō;

El si ečava en la kama,

I en el terēnu yō.

Al fin di medža noči

Al agwa mē mandó:

Al suniziku ${ }^{8}$ de la agwa

Durmyēra mi yō.

1 S. Sois.

2"Left."

3 "To tie."

4! Another version is given in Danon's collection.

5 S. juntos.

6 "Because."

${ }^{7}$ S. huesecico.

8 "Murmuring." 
Por ayi pasó un kavayēru,

Trēs bizikus mi dió.

Gway di mi, dizvinturada,

Kē pekadus fizyēra yō?

Si mi maridu lu savi,

Matada seré yō!

Antis ke el mi mati,

Matarmi këro yō.

"Ni vos mateš, mi siñora,

Ni teneš porké vos matar,

Kē yō so vwestru maridu,

Vwestru riku pastor !"

$\mathrm{X}$.

Mi madri salyó a la luna

Por ver mi bwena vintura

En el lunar,

Kē la luna al kavayēru

A medža noči al bel lunar.

Mi madri salyó a la estrēya

Por ver mi bwena planeta

En el lunar, etc.

"Nōn es, mi madri, la luna,

Sinōn mi bwena vintura

En el lunar, etc."

"Mentideš, fiža, mentideš,

Kē la toka yō lē vidi

En el lunar, etc."

"Nōn es, mi madri, la toka,

Sinōn mi kara redōnda

En el lunar, etc."

"Mentideš, fiža, mentideš,

Kē la espada yō lē vidi

En el lunar, etc."

"Nōn es, mi madri, la espada,

Sinōn mi bella garganta

En el lunar, etc."

"Mentideš, fiža, mentideš,

Kē el kavayu yō lē vidi

En el lunar, etc." 
"Nōn es, mi madri, el kavayu,

Sinōn mis bellus trēnsadus

En el lunar, etc."

"Mentideš, fiža, mentideš,

Kè al ladu volu vidi

En el lunar, etc."

"I si milu viteš al ladu,

Mi padri milu merkó

Por un dukadu

En el lunar, etc."

XI. ${ }^{1}$

Alkansar al kavayēru,

Alkansar komu sayeta,

En lugar di ir a la karsil

Ondi la rēna se ia.

Topó la rēna en kavēyus,

En kavēyus i trēnsadus,

Peñandu lus sus trēnsadus

Kon un peñi di oru maru, ${ }^{2}$

I un espežu muy lindu,

Kē en el resta su peñadu,

Dandu loōris al Dyó altu,

Kè tan linda la a kreadu.

El rē por burlar kon eya

Kon el verdugu lē a dadu.

"Stati, stati, Andarlētu,

Mi pulidu inamoradu!

Dōs fižikus di ti tengu

I dōs del rē, kē son kwatru.

Lus del rē suvin kavayu,

I lus tuyus mula i kavayu;

Lus del rē komin en mēza,

I lus tuyus al mi ladu;

Lus del rē van a la gēra,

I lus tuyus al mi ladu;

Lus del rē yevan espada,

I lus tuyus spada i endoradu. ${ }^{3}$

Mas ti kēro i mas ti amo

Kē non al rē kon su rēnadu:

El rē mi dava dinerus

1 Another version is given in Danon's collection.

2 "Pure."

$3(?)$ 
I tu mi davas dukadus."

Boltó su kara a la rēna,

Topó si al rē al ladu.

"Perdon, perdon, siñor rē!

Todu estu kē yō a favladu

Kē a noči, a medža noči,

Todu estu melu a soñadu."

"Ya telu perdoní, la rēna,

Kon la kavēsa a un ladu."

Mandó a yamar a Andarlētu,

Su pulidu inamoradu.

"Komu farēmus, Andarlētu,

Kē el rē mus a aminazadu?

Para mi topí remedžu,

Para vos, andá i bušká vus!"

A mal vaygan las mužeris

Kē en ombri si an kunfiadu :-

Tenyendu al rē por maridu

$\mathrm{X} w e$ a buškar inamoradu.

"Mas ti kero i mas ti amo

Kē non al rē kon su rēnadu!"

Estas palavras dizyendu

La kavēsa lē a kortadu;

Estas palavras favlandu

La kavēsa lē ečó a su ladu.

\section{XII. ${ }^{1}$}

Yō kaminí por altas maris, Navigi por las fortunas, Kayí mi en sivdat ažena, Ondi nōn mi kunosian.

Fayarun mi dōs dženeralis,

Lus mas grandis di Turkia:

"Kē buškavaš vos, buen mučaču?

Kē buškavaš por estas vias?"

"Buško yō al rē mi padri,

La korona kē tenia."

"Una vēs kē tu lu buškas,

Kē señas di el darias?"

"Añus tenia sesenta,

La barva blanka tenia."

"Una vẽs kē tu lu buškas,

1 Another version is given in Danon's collection. 
El rē turku lu mataria."

Tomó lu manu por manu,

A mostrar selu iria:

Yevó mi en un monti iskuru,

Kē di negru paresia.

Razgósi el lus sus pañus

Di sayo asta kamiza.

Asta akí es el romansu.

$\mathrm{Su}$ alma en folgansa seria.

\section{XIII. ${ }^{1}$}

"Morenika mi yama

El fižu del rē;

Si otra ves mi yama

Yō mi vo kon el.

"Morenika mi yama,

Yō blanko nasí;

Di pasear galana

Mi kolor perdí."

"Abašeš, morēna, Si aveš di abašar!

Kē la navi tengu en vēla,

Mi kēru andar."

Eya si viste di verdi

I di amariyu,

Kè ansi dizi la pēra

Kon el bimbriyu. ${ }^{2}$

Eya si viste di verdi

I di zurzuli, ${ }^{3}$

Kē ansi dizi la pēra

Kon el čufteli. ${ }^{4}$

Eya si viste di verdi

I di otru kolor,

Kē ansi dizi la roza

Kon el ambimbroy. ${ }^{5}$

De la mar abašu

Lu vidi venir:

"Kē aki yō vus asperu

A dar kidušin. ${ }^{6}$

1 Another version is given in Danon's collection.

2 "Citron melon."

3"Yellow peach."

4 "Peach."

3 Some flower (?)

$6 \mathrm{H} .=$ "betrothal." 
De la mar abašu

Lu vidi abašar,

Kē akumpañadu vyeni

A dar irusin. ${ }^{1}$

"Abašeš, morēna,

A la xwenti ${ }^{2}$ alta,

Kē toda la kē la vei

Keda preñada!

"Abašeš, morēnā,

A la xwenti di ariva

Kè toda la kē la bēvi

Vyeni parida!"

\section{XIV.}

En la mar batin las olas,

Las mučačas durmin solas-

Si kereš saver, Bulisa,

La kantiga de las oras.

A tan alta kè va la luna.

A tan altu kē va al sol,

Kwandu durmi la kriatura,-

Si kereš saver, Bulisa,

La ora ya tañi una.

Di kantar ya no mi keda boz,

A la gwerta yevan el aroz,-

Si kereš saver, Bulisa,

La ora ya tañi dos.

A Istambol mi keria ir a ver,

Mi kuntarun kē es bwen a ver,-

Si kereš saver, Bulisa,

La ora ya tañi tres.

En Belugradu no ay garatu, ${ }^{3}$

El vinu bēvin baratu,-

Si kereš saver, Bulisa,

La ora ya tañi kwatru.

Al čurčiku* lu yaman riku,

Mi kuntarun kē es muy riku,--

Si kereš saver, Bulisa,

La ora ya tañi sinku.

$1 \mathrm{H}$. = "mode of betrothal."

$2 \mathrm{~S}$. fuente.

3 Some fish (?)

4(?) 
En el xan ${ }^{1}$ ay una kubé, ${ }^{2}$

Lus morus lu yaman meš,-

Si kereš saver, Bulisa,

La ora ya tañi seš'.

Di alavar a lus mis paryentis,

En la bōka no mi kedó dyenti,-

Si kereš saver, Bulisa,

La ora ya tañi syeti.

Peši fritu i muy byen koču, Por mezध ${ }^{3}$ un bwen bizkoču,

Si kereš saver, Bulisa,

La ora ya tañi oču.

El mundu si aribolvi,

La preñada a lus mezis muēvi,-

Si kereš saver, Bulisa,

La ora ya tañi muēvi.

En la mēza kē ay muēzis, ${ }^{4}$

El vinu bēvin todus lus mēzis,-

Si kereš saver, Bulisa,

La ora ya tañi dyes.

La uyika ${ }^{5}$ buyi i kozi,

Abašalda kē si aripozi,-

Si kereš saver, Bulisa,

La ora ya tañi onzi.

Aydi, aydi, kē es di noči,

En Yirušalaim bēvin leči,-

Si kereš saver, Bulisa,

La ora ya tañi dodzi.

\section{$\mathrm{XV} \cdot{ }^{6}$}

Raxel, mwesträ maðri, asuvyó a lus syēlus,-

Intanid in bokä avló kun el Viðraderu. ${ }^{7}$

Mirä kē alegriä ay en Istrael,

K' el Dyó de lus syēlus kē mulu deše ver.

En la kazä santä ay un almenarä,

Syeti brasus tyeni k' al mundu interu arelumbrava.

El beðamigdaš ${ }^{8}$ lu stan fragwandu,

Kon pyeðräs presyozäs lu stan aruðyandu.

1 T. = "inn." $\quad 2(?) \quad 3$ "Lunch." $\quad 4$ S. nuez. $\quad 5$ S. ollica.

6 Another version given in Danon's collection. $\quad 7 \mathrm{~S}$. verdadero. $\quad 8 \mathrm{H} .=$ " temple." 
Xaxamím ${ }^{1}$ kantavän; lus Leviím ${ }^{2}$ baylavän, I lus Koaním ${ }^{3}$ karbán $^{4}$ ayigavän. ${ }^{5}$ Ay en el miðbar ${ }^{6}$ tres palombäs bolän,Malaxím ${ }^{7}$ del syēlu avlavän bokä kun bokä.

XVI.

Sinku añus ya v' azer Ki yo tengu displazer Kun un mansevu d' Izmir,

Ki yo pur el mi vo murir,Noči i dia kun pasyô Azyendu la orasyô, Arugandu al Kriaðor Ki mi skapi d'est' amor. Intri gwertäs i veryelis Yo bivir kun doloris Un fistan mi vo kurtar,Gway', ki no lu vo gozar! Unä kartä vo skrivir A la mi mamä suvenir. Ki la meldi i ki yori Ki s' akoðrä ${ }^{9}$ di mi. Kazaminterus veyo venir Parä dispuzarmi a mi. Estu no lu pweðu sintir, Ki aboltin lakardí! ${ }^{10}$ Unä kartä vo skrivir A lus mansevus suvenir, Ki no atin mas amor Ki l'amor azi puðrir. Yo. kwandu mi vo murir, En la pyeðrä vo skrivir, $\mathrm{Ki}$ la meldin i ki yorin, Ki l'amor azi puðrir.

\section{XVII.}

Uyí dizir kē boðås ay pur akí, kē vengu a ver, Kē gozi i kē logri i kē tengä munču byen! Uyi dizir, in esti palasyu kē gozi la novyä in un mazal $^{11}$ muy klaru,-kazamyentu kē del Dyó es,-el novyu no keri

\footnotetext{
$1 \mathrm{H}$. = "wise men."

2 H. = "Levites."

3 H. = "Cohanites."

$4 \mathrm{H} .=$ " treasure."
}

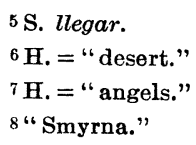

${ }^{9} \mathrm{~S}$. acordarse.

10 Conversation.

11 H. = "luck." 
munčus dukaðus, keri a la novyä in mazal klaru. El novyu no keri dinerus, keri a la novyä in un mazal muy bwenu. Oy kazavä la blankä niña kun un mansevu d'estäs viyäs,--kazamyentu del Dyó es,--kun un mansevu koma la rozä,--Syen añus kē turi dičozä.

\section{XVIII.}

Un bwer ${ }^{1}$ rey está xazinu, I amandän pur lus doktoris, Kwantus pur el mundu sô. Unus enträn i unus salen,Ningunu no l' aprovečo.

I amandän pur el mas grandi, El mas grandi i el mayor.

A la suviðä del' eskalerä Di roðiyäs si suvyó;

Al' entraðä de la pwertä

La kavesä li kayó.

S' asintí a la kaviserä, El pulsu li atintó.

Le preguntó el xazinu al doktor, Ke tal li paresi.

Li dišo el doktor:

“D' esti mal kē tengu yo!"

S'abultó del otru kavu el xazinu, Dišu el xazinu:

“ Unus goðän las mužeris, Yo pur gozar la vo dešar; Unus goðän sus kriaturäs, Yo čikus lus vo dešar." Estäs palavräs dizyendu, Er rey se akavó,Er rey di primä noči, El doktor a mañanä al alvor.

\section{$\mathrm{XIX}^{2}$}

Al kinzi del mes la lunä en kresyenti, Kwandu er rey Nemrod salyó estreyeru, El indivinó kē nasia Mošé.

La mužer di Terax preñadä stavă, Den dia en dia le dimandavä:

De kē yevaš la karä tan dimuðaðä?"

Eya si saviä el mal kē teniä,- 
Si salyó pur lus kampus koma peðriðã, Doloris teniä i parir keriä,--

Andi lu paryerä in la meará.

Al kavu di oču dias lu xwé a buškar, Andi lu tupavä in la yešivá. ${ }^{2}$

Kwandu nasyó 'l ižu, arilumbravä

Komu 'l sol i la lunä a meðyu dia.

Kwandu nasyó 'l ižu, in lugo ${ }^{3}$ avlavă:

"Indá ${ }^{4}$ us, la mi maðri al vwestru lugar,

Ke yo ya tengu aličaðeräs,

I yo ya tengu miraðeräs, kē mi mirarán."

Gran zaxut ${ }^{5}$ tuvitiš, siñor di Avraam,

Kē kun su manu mizma s'izu birmilá! ${ }^{6}$

Gran zaxut tuvitiš, siñor pariðu,

$\mathrm{K}^{\prime}$ afirmatiš la mítsva ${ }^{7}$ d'Avraam avinu. $^{8}$

\section{XX.}

Estäs mezăs son del vinu,-

Bivå la patronä kun su mariðu!-

Estäs mezăs mezăs sô.

Estäs mezäs son del klaru,

Bivă la patronä kun su amaðu,

I las kē stan al deredor!

Estäs mezäs mezäs sô.

"S'aveš kumiðu koma 's la razon,

La patronä de la kazä muz dimandä piðron." 10

“ No kumimus naðä, lindä kumpañă,

No kumimus naðä, lindä kumpañã,-

Beraxa ${ }^{11}$ mus aga!"

“ $S$ ' aveš kumiðu koma 's el dever,

La patronä de la kazä muz dimandä mersé."

"No" kumimus naðä, etc.

“ $S$ ' aveš kumiðu peši de la mar,

Nainda sta pur alkansar,--

El peši sta pur alkansar,

La reynä sta pur enreynar."

$1 \mathrm{H} .=$ "cave."

$2 \mathrm{H}$. = " house of study."

$3 \mathrm{~S}$. luego.

4 S. andad.

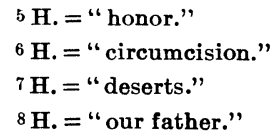


“ No kumimus naðä, etc.

" Kun vrigwensä ${ }^{1}$ vu lu diku, Kē ya mi vo kun mi amigu. Kun vrigwensä vu lu avlu, Kē ya mi vo kun mi amaðu."

“Iža, mi vayaš en la oră bwenă!"

\section{XXI.}

Amor tengu, no paresku,

Ni me do pur konsintir,-

El la pwertä di la kayi

L'alma ya mi va salir.

Tres añus d'amor ki ize,

Al kwartér mi izvačií. ${ }^{2}$

Añu i meðyu di kazaðã

Ufisyu no li tupí,--

El ufisyu del mi mariðu

Es ladron i kumardží. ${ }^{3}$

El Taván ki mi lu gwaðri ${ }^{5}$

Di la manu del polís. ${ }^{6}$

Pretä se yo, la mi maðri,

Ni si tomi sixurá: ${ }^{7}$

Lus ožus di lus mansevus

Ya si inčin kun parás.

\section{XXII. ${ }^{8}$}

Morenä mi yamän

Yo blankä nasí.

Di pasiyar galana

Mi kolor piðrí. ${ }^{9}$

Morenä mi yamå

El ižu di alvor;

Si oträ ves mi yamå

Yo kun el mi vo.

D'akeyäs vintanäs

M'arondžan ${ }^{10}$ flečäs;

Si sô di amoris

Vengän derečäs!

1 S. virguenza.

2 T. = " to resign."

4 T. = "god."

5. guardar.

8 Another version given in Danon's collection.
6 "Police."

9 S. perder.
3 $\mathbf{T} .=$ "gambler."
7 "Care." 10 "Throw."




\author{
D’akeyäs vintanäs \\ M' arondžan arkus; \\ Si sô di amoris \\ Vengän al laðu! \\ Vestiðä di veðri ${ }^{\perp}$ \\ I di altelí,-2 \\ K’ansi ${ }^{3}$ dizi la novyä \\ Kun su čilibi. ${ }^{4}$ \\ Eskalerikä l’izi ${ }^{5}$ \\ Di oru i di marfil, \\ Kē vengä la novyä \\ A dar kídušín! \\ Eskalerikä l'izi \\ Di oru i di perlä, \\ Kē vengä el novyu \\ A dar beraxát!
}

\title{
XXIII.
}

Kwandu Mošé Rabenu ${ }^{6}$ kižu $^{7}$ êrreynar ${ }^{8}$

Lus syēlus i la tyerä izu temblar.

Mirá, kē siñor erä Mošé Rabenu,

K'asuvyo i abaš́ a lus syēlus!

Un prezenti tengu, lu vengu a tomar, K' èrä la ley santã i el sefér-torá. ${ }^{9}$

Un prezenti tengu, lu vengu a resivir K' èră la ley santä i el sefér-šeli. ${ }^{10}$

Mirá, kē siñor erä Moš́́ Rabenu, K' abašavä i asuviä a lus syeti syēlus.

XXIV.

Pariðä, el Dyó vus gwaðri, ${ }^{11}$

Kwantu keri la vwesträ madri, Di toðu el mal!

Pariðă, el Dyó vus deši, Kwantu keri la vwesträ dženti,

Di toðu el mal!

$1 \mathrm{~S}$. verde.

2 Some color.

$3 \mathrm{~S}$ que asi.

$4 \mathrm{~T} .=$ "lover."

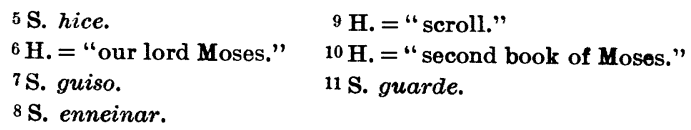


Pariðã stavä la dweñä i pariðä soš!

Kè byen empleyaðäs xwerun las doloris

Kē nasyó un ižu di beyäs ${ }^{1}$ koloris!

Syempri de kontinu a el Dyó daremus las loris. ${ }^{2}$

Ya vyeni el pariðu kun sus manus yenäs

En la unä manu un masu di kandeläs,

En la otră manu mansanäs i perăs!

Kwandu la komaðri dizy'a la pariða: "Haydi, haydi!" ${ }^{3}$

Rispondi la pariðä: "Adunay, ${ }^{4} \mathrm{ke}$ mi skapi!"

Ya vyeni el pariðu al dib ${ }^{5}$ de la kamă,

Dizya la pariðă: "Lu kē keris, mi almä?"

Rispondi la pariðä kē esta bwenä.

\section{XXV.}

Un riyo d'unä fwenti, Unä saká ${ }^{6}$ lavi;

Al ruiðo del agwä

Yo mi aserki ast' ayí.

Sintí unä boz kē diziä:

"Ay de mi, ay de mi, ay de mi!"

Si komu mi aserki a su laðu

Beðyäs ${ }^{7}$ floris kurtí

Dispwes dixú ${ }^{8}$ la niñã

I akayó, i akayó, i s' ezmayó.

Si komu la viðé sulikä

Li diklarí mi amor;

Yurand ${ }^{9}$ mi dizia:

"No t' ulvides tu del Dyo."

Dispwes dixú la niña, etc.

Si komu la viðé sulikä

Al kafé la yeví;

Li divizi lu pretu,

Tres bezus l' estampí

Dispwes dixú la niña

Otrus tres, otrus tres, s' azin seš.

1 S. bellas.

s. loores.

3 Turkish greeting.

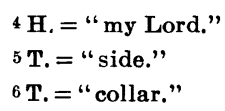

272
7 S. varias.

8 "Fainted."

9 S. llorando. 
Al despartirme d'eya

Un abrasu mi diyó

Non si viðo muriente

Non si viðo muriente

Dispwes dixú la niña

I akayó, i akayó, i s’ezmayó.

\section{XXVI. ${ }^{1}$}

Nočis bwenäs, nočis bwenäs,

Nočis sô d' inamorar;

Nočis sô d' inamorar,

Dandu bweltäs pur la kama

Koma 'l peškaðu en la mar.

Estä noči, la mi maðri,

No la puðe sinportar:

Luvyäs kain di lus syēlus,

Lagrimäs di mis ožus.

Tres ermanikäs erån,

Toðăs tres a un metá;

Saltó la primerä d' eyäs,

Maðri miya, la mi keriða,

Nunkä no va ver kazar.

\section{XXVII.}

Unä noči al lunar

Mi salí a pasiyar.

Un mansevu mi topó

Al gazinu mi yevó.

El la siya m' asentó

Ună biră mi dyó.

Mi dimand' a caz' aparte

Vintanäs para yalí; ${ }^{2}$

Mi dimandä unås dimandäs

Kē mi azin tresalir:

"Yamaremus Xazaním ${ }^{3}$

Kē mus den lus kidušín!”

\section{XXVIII."}

Andandu pur estås maris

Navigí kun grandi fortună,

1 R. d. E. J., Vol. XXXIII, p. 119, has a variant of this.

$2 \mathbf{T} .=$ "seashore."

$3 \mathrm{H} .=$ "cantors." $\quad 4$ R. $d . E . J .$, Vol. XXXIII, p. 115, has another variant of this. 
Kayí in tyeräs aženäs

Andi no mi kunusian

Andi no kantavä gayo

$\mathrm{Ni}$ la leonä arespondiä.

Lagrimäs di mis ožus

Barru l' amasaré;

Kun las plantäs de las manus

El barru lu embarraré;

Kun suspirus di su almä

El barru lu enšugaré.

Toðu ombri k'es kaminanti

Kun mi lus tumaré.

El ki konti di sus malis

Di lus miyus yo li kuntaré.

Si lus suyus sô mas munčus

A pasyensyä mi lus tumaré.

Si lus miyus sô mas munčus

A pasyensyä mi lus tumaré.

Leo Wiener.

HARVARD UNIVERSITY. 